\title{
How accurate do x-ray data need to be to obtain a reliable order of stability for polymorphs? The case study of $p$-hydroxyacetophenone polymorphs
}

\author{
Marcin Sztylko, Maura Malinska, Vaclav Petricek, Matthias J.
} Gutmann and Anna A. Hoser

\section{Published version information}

Citation: M Sztylko et al. "How accurate do x-ray data need to be to obtain a reliable order of stability for polymorphs? The case study of $p$-hydroxyacetophenone polymorphs." Crystal Growth \& Design, vol. 19, no. 9 (2019): 5132-5141.

DOI: $10.1021 /$ acs.cgd.9b00518

This document is the unedited author's version of a Submitted Work that was subsequently accepted for publication in Crystal Growth \& Design, (C2019 American Chemical Society, after peer review. To access the final edited and published work see DOI above.

Please cite only the published version using the reference above. This is the citation assigned by the publisher at the time of issuing the AAM. Please check the publisher's website for any updates. 


\section{CRYSTAL GROWTH हुDESIGN}

Subscriber access provided by The Chadwick \&amp; RAL Libraries

\section{Article}

\section{How accurate X-ray data are needed to obtain reliable order of stability} for polymorphs? The case study of p-hydroxyacetophenone polymorphs.

Marcin Sztylko, Maura Malinska, Vaclav Petricek, Matthias J Gutmann, and Anna A. Hoser Cryst. Growth Des., Just Accepted Manuscript • DOI: 10.1021/acs.cgd.9b00518 • Publication Date (Web): 22 Jul 2019 Downloaded from pubs.acs.org on August 6, 2019

\section{Just Accepted}

"Just Accepted" manuscripts have been peer-reviewed and accepted for publication. They are posted online prior to technical editing, formatting for publication and author proofing. The American Chemical Society provides "Just Accepted" as a service to the research community to expedite the dissemination of scientific material as soon as possible after acceptance. "Just Accepted" manuscripts appear in full in PDF format accompanied by an HTML abstract. "Just Accepted" manuscripts have been fully peer reviewed, but should not be considered the official version of record. They are citable by the Digital Object Identifier (DOI®). "Just Accepted" is an optional service offered to authors. Therefore, the "Just Accepted" Web site may not include all articles that will be published in the journal. After a manuscript is technically edited and formatted, it will be removed from the "Just Accepted" Web site and published as an ASAP article. Note that technical editing may introduce minor changes to the manuscript text and/or graphics which could affect content, and all legal disclaimers and ethical guidelines that apply to the journal pertain. ACS cannot be held responsible for errors or consequences arising from the use of information contained in these "Just Accepted" manuscripts. 


\title{
How accurate $X$-ray data are needed to obtain reliable order of stability for polymorphs? \\ The case study of $p$-hydroxyacetophenone polymorphs.
}

\begin{abstract}
Marcin Sztylko , Maura Malinska1, Vaclav Petricek², Matthias J. Gutmann³, Anna A. Hoser $^{1 *}$

${ }^{1}$ Biological and Chemical Research Centre, Faculty of Chemistry, University of Warsaw, Żwirki i Wigury 101, 02-089 Warszawa, Poland

2Institute of Physics, Academy of Sciences of the Czech Republic, Na Slovance 2, 18221 Praha, Czech Republic

${ }^{3}$ ISIS Neutrons and Muons Facility, Science and Technical Facility Council, Rutherford Appleton Laboratory, Harwell Science Campus, Didcot, OX11 OQX, UK
\end{abstract}

* Corresponding author: Anna A. Hoser (annahoser@chem.uw.edu.pl)

\begin{abstract}
:
Technical progress in crystallographic instruments allows contemporary crystallographers to more routinely conduct excellent quality low-temperature diffraction measurements. As a result, even crystal structures that have been extensively studied at ambient conditions start to reveal their more complex nature. The importance of low-temperature measurements is discussed based on the new, modulated structures found for polymorphs of 4'-hydroxyacetophenone (HAP). Diffraction data for this polymorphic system have been collected in the temperature range temperature range from $40 \mathrm{~K}$ to $330 \mathrm{~K}$. At $123 \mathrm{~K}$ form II of HAP appears to be a new, commensurately modulated structure with a modulation vector $q=[0.4,0.0,0.0]$. The structure can be solved and refined with 10 molecules in the asymmetric unit. Further decrease of temperature to $40 \mathrm{~K}$ resulted in a discovery of a new, incommensurately modulated phase with four independent molecules in the P21 monoclinic space group with modulation vector $\mathrm{q}=[0.37,0.17,0.0]$. Consequences of modulation for the relative stability of polymorphs will be discussed.
\end{abstract}

Keywords: 4-hydroxyacetophenone, polymorphs, relative stability 


\section{Introduction}

Polymorphism is the ability of a solid material to exist in more than one form of crystal structure. As it was shown in many cases, different polymorphic forms can exhibit different physicochemical properties and bioactivity. Around 30\% of drugs exist in more than one polymorphic form ${ }^{1}$, and as those forms may differ in solubility and other important physicochemical properties, it is crucial to identify and characterize all polymorphic forms. Moreover, it is desired to gain knowledge about relative stability of polymorphic forms of a given substance. Experimentally, the relative stability of polymorphs can be obtained from calorimetric measurements or from solubility measurements.

There are also many theoretical studies which are focused on investigating relative stability of polymorphic structures, showing that differences in Gibbs free energies between polymorphic forms can be extremely small. For example, according to study by Nyman and Day conducted for 508 organic polymorphic systems over half of polymorph pairs are separated by less than $2 \mathrm{~kJ} / \mathrm{mol}^{2}$. Therefore, if a given theoretical method is capable of predicting the relative stability in agreement with experiment for a wide range of polymorphic systems such a method should be considered as highly accurate.

Very often, starting point for such calculations are structures obtained from the Cambridge Structural Database (CSD), which are subsequently optimized. In such a case it is important to know, whether a given structure contains disorder or modulation, as all those features affect crystal stability. Up to now, anisotropic atomic displacement parameters (ADPs) are not stored in the official release files, therefore it is difficult to judge the quality of the structure.

This leads to a few important questions: How good X-ray data do we need in order to use them as an input for calculations of relative stability of polymorphs? How subtle structural details one needs to know for the studied polymorphic structures? And how many of those subtle details are overlooked in data deposited in the CSD?

In 2001 the CSD contained around 250,000 structures. Currently, in June 2019, the CSD has reached 1,000,000 structures. Nowadays X-ray diffractometers are much more advanced than 20-30 years ago. They have higher brilliance sources, micro sources, 
rotation anodes, and more sensitive faster 2D detectors. Moreover, there is a huge progress in data reduction and integration software as well. It has several consequences: we can collect data faster to higher resolutions than it was possible 30 years ago, and we can process them better. Additionally, there are several charge density models available, among them Transferable Aspherical Atom Model (TAAM) ${ }^{3,4}$ or Hirshfeld Atom Refinement (HAR) ${ }^{5-7}$ that exceed the accuracy of the widely used Independent Atom Model (IAM). All of the above models can be applied during data refinement in order to obtain geometry parameters closer to optimized or neutron structure. Therefore, in principle, XRD data collected now, should be more accurate than those collected 20-30 years ago. Unfortunately, it doesn't necessarily mean that data collected now and deposited in the CSD are more accurate, and there are several reasons for it.

First of all, software currently used for data reduction and integration is automated, to such a degree that one may solve and refine structure without even looking at diffraction frames. In consequence, as was already discussed by Andrew Bond ${ }^{8}$, many structures are measured, solved and refined by researches with limited experience in crystallography. As a result, some effects, such as diffuse scattering, disorder, or modulation, might simply be overlooked.

Secondly, even if crystal quality allows for a collection of high resolution, it is not done due to the lack of time. Unfortunately, common practice is to cut the data to lower resolution in order to obtain better statistics after refinement. In consequence, some subtle, but still important, features might be missed.

Thirdly, an important issue is the temperature of the data collection. More than a half of structures, deposited in the CSD were measured at room temperature only. As at room temperature it is difficult to obtain high resolution $\mathrm{X}$-ray data for organic samples small disorders or modulations can be neglected due to high temperature factors. An interesting system, for which we already show, how important it is to conduct measurements not only at room temperature is gallic acid monohydrate ${ }^{9}$. A polymorph of GAM was the target of structure prediction in the fifth blind test (CSP target XXI, CSD reference codes KONTIQ037 and KONTIQ056), yet it was the only system that could not be predicted within the three most likely structures by any of the fourteen participating research 
groups. The measurements, which were conducted later on, demonstrate that the structure is commensurately modulated at $10 \mathrm{~K}$ and disordered at higher temperatures, and that disorder gives substantial stabilization of the structure.

Therefore, we may claim that disorder, higher Z', and modulations will contribute to Gibbs free energies of given polymorphic forms. In the case of polymorphs, where differences between both forms are below a few $\mathrm{kJ} / \mathrm{mol}$, neglecting such subtle structural details in the process of prediction of phase transition temperature may lead to incorrect stability order at given conditions.

In this contribution we will focus on the results obtained for a small, rigid system, namely 4'-hydroxyacetophenone (HAP). Up to now, there are two known polymorphic forms of HAP. Both forms crystallize concomitantly, and according to calorimetric study by Bernardes et al. ${ }^{10}$, they are enantiotropically related - phase transition from stable at low temperature form II to form I occurs at around $351 \mathrm{~K}^{10}$. Recently, the phase transition temperature was estimated from adiabatic calorimetry measurements to be around 300 $\mathrm{K}^{11}$. In the CSD, there are deposited structures of HAP polymorphs from $150 \mathrm{~K}$ and $298 \mathrm{~K}$. Herein, we will present structures of HAP measured below $150 \mathrm{~K}$. Our investigations demonstrate that form II of 4'-hydroxyacetophenone at $100 \mathrm{~K}$ is commensurately modulated and after lowering temperature to $40 \mathrm{~K}$ we observe that structure is incommensurately modulated. X-ray and neutron diffraction data for both forms were collected in $100 \mathrm{~K}$. For form II of HAP weak peaks from modulation are observed from neutron diffraction data, similarly as from X-rays. Later on, we will discuss importance of the ADPs, as their direction and size at room temperature can indicate some interesting effects, such as modulations, disorders occur when the temperature will be sufficiently low. At the end, we will try to answer the question: is it really crucial, to be aware of such small structural differences between polymorphs?

\section{Methods}

\subsection{Crystallization}

Crystals of form I were grown by slow evaporation of solvent from a saturated ethanol solution of this substance over the course of several days at the room temperature $\left(23^{\circ} \mathrm{C}\right)$. form II was crystallized according to the procedure reported by Kulkarni et al ${ }^{12}$. Slow 
evaporation at room temperature from the ethyl acetate solution at a concentration below $250 \mathrm{mg} / \mathrm{mL}$ was performed in a small sample vial. After approximately 48 hours crystals of good quality and suitable size were chosen for X-ray diffraction measurements.

\subsection{Single crystal X-ray diffraction measurements}

Single crystal X-ray diffraction data of form I and II were collected on the Agilent Technologies SuperNova diffractometer with MoKo source at 40K, 100K, 123K, 150K, 200 $\mathrm{K}$ and room temperature. Additionally, for form II we collected data at 330K. Data reduction was done using CrysAlis RED software ${ }^{13}$. The structure was solved with direct methods and then successive least-square refinements were carried out based on fullmatrix least-squares on $\mathrm{F}^{2}$ using the SHELX program ${ }^{14}$. Structures are deposited in the CSD, with deposition numbers CCDC 1910394-1910408. The modulated structures were refined in JANA program package ${ }^{15}$. The structure from $40 \mathrm{~K}$ was integrated as pseudomorohedric twin related by two-fold axis along the direction [ 100 l 0 . For structure refinement the model found for the $100 \mathrm{~K}$ phase was used as a starting point after transformation to the subgroup P21. As the number of observed reflections is extremely small, we have used a rigid body approximation of the HAP molecule to describe four molecules in the asymmetric unit. For crystallographic details see Table 1 in Supporting Materials. As it was shown that from TAAM refinement it is possible to obtain more accurate atomic positions and ADPs than from routine IAM ${ }^{16}$, TAAM refinements were conducted using the MoPro package ${ }^{17}$. Multipole population parameters were derived from the $\mathrm{UBDB}^{3}$ with the aid of the LSDB program ${ }^{4}$. Thermal expansion plots were generated in PASCal program ${ }^{18}$.

\subsection{Neutron diffraction measurements}

The neutron experiments were performed on the Time-of-Flight (TOF) single crystal Laue diffractometer (SXD) ${ }^{19}$ at ISIS (Chilton, UK). The data were collected at $100 \mathrm{~K}$. The integration process was carried out with the SXD200120 program. The structure was then refined using JANA with the single batch of wavelength and extinction-corrected reflections ${ }^{15}$. 


\subsection{Computational details}

\subsubsection{Energy Frameworks}

Interaction energy between molecular pairs has been calculated with CE-B3LYP/6$31 \mathrm{G}(\mathrm{d}, \mathrm{p})$ model implemented in CrystalExplorer1721, 22. This allowed for a construction of energy frameworks, which display pairwise energies in the crystal as cylinders proportional in size to the magnitude of the interaction energy. All calculations were based on experimental structures deposited in the CSD (deposition numbers CCDC $686778-686781)$.

\subsubsection{CRYSTAL17 computations}

\section{Lattice and total energies}

All calculations of lattice energy were performed with a CRYSTAL17 code ${ }^{23,24}$. The structures were optimized with the dispersion corrected PBE0-D3(BJ) hybrid functional25 and the TZP basis set developed by Ahlrichs and co-workers ${ }^{26}$. The unit cell parameters were fixed with lattice parameters determined from the X-ray diffraction experiments at a given temperature, allowing only the atomic coordinates to vary during optimization. We tested the performance of PBE0 functional in combination with two dispersion correction schemes - D2[5] and D3(BJ) ${ }^{27,28}$. To further validate our results we ran calculations at the B3LYP-D3ABC/TZP level of theory on the dispersion-scaled HF-3c optimized geometries, an approach recently proposed by Ugliengo and co-workers ${ }^{29}$.

\section{Frequency calculations and ADPs estimation}

Similarly to lattice energy calculations, geometry optimization (only atomic coordinates) and $\Gamma$ point frequency calculations were conducted in CRYSTAL17. Calculations were performed with B3LYP 30 functional and 6-31G(d,p) basis set. Obtained frequencies were utilised to calculate ADPs at $100 \mathrm{~K}$, as it was proposed by Madsen et al. ${ }^{31}$ 


\subsection{Results and discussion: Structures from X-ray diffraction data and Energy Frameworks}

Structures of both polymorphic forms of HAP have been previously described ${ }^{10}$. We provide a table with all crystallographic details from our experiments and detailed in the Supporting Information.

Form I and II of HAP are conformational polymorphs. In respective polymorphic forms molecules of HAP differ mainly in the orientation of the hydroxyl group with respect to the carbonyl group. In the monoclinic form I there is only one molecule in the asymmetric unit, which adopts E conformation (anti), whereas in form II, there are two molecules that both adopt $\mathrm{Z}$ conformation (syn).
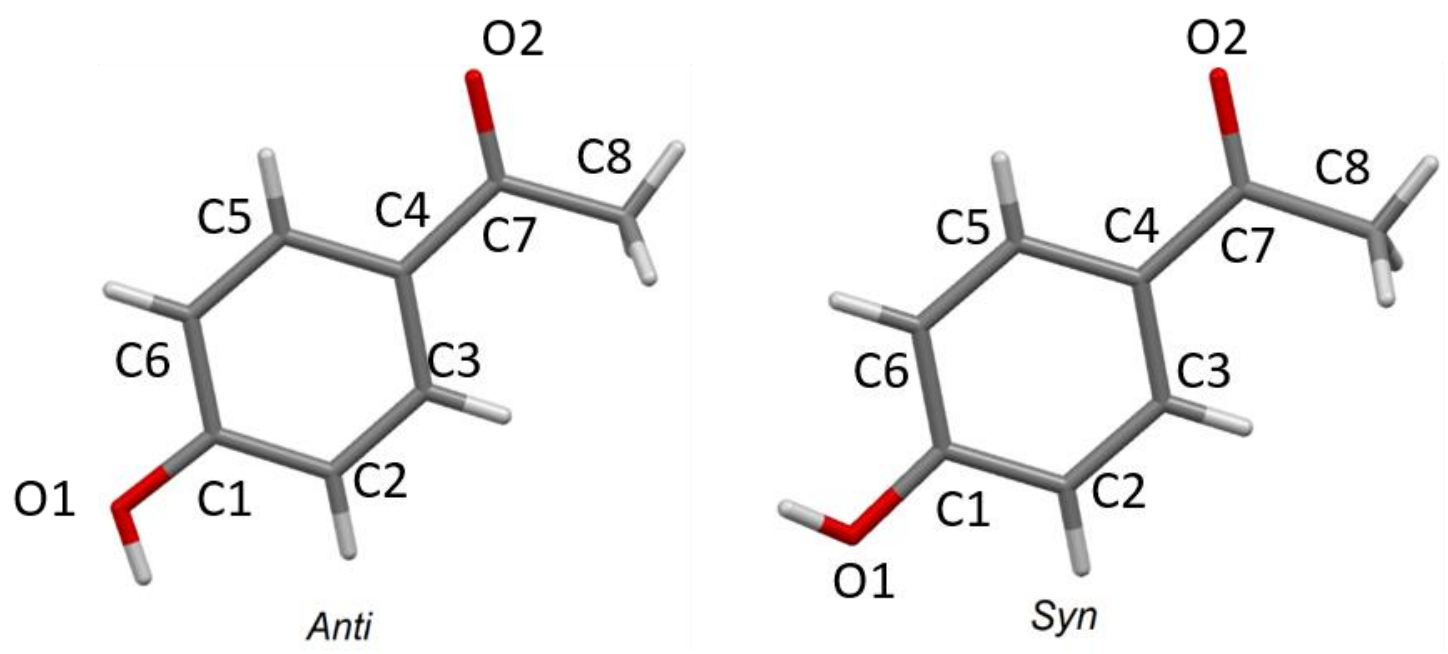

Figure 1. Molecular conformations of HAP form I (anti) and HAP form II (syn)

Despite the difference in conformation, both polymorphs form "head-to-tail" chains via hydrogen bonding between the hydroxyl group of one molecule (donor) and the carbonyl group of an adjacent molecule (acceptor). However, the different conformation of hydroxyl group leads to either linear chains (form I) or helical chains (form II).

To better understand structural differences in this polymorphic system we used the energy framework analysis ${ }^{22}$. This tool helps to visualize interaction energies governing molecular packing in the crystal lattice. In the case of HAP polymorphs the main structural motif, i.e. chains of hydrogen bonds, is clearly reproduced through a coulombic interaction (see Figure 1). In both polymorphs, hydrogen bonded chains in third direction are connected via dispersion interactions. However, the precise values of interaction energies 
reveal the differences between the two polymorphs. In the HAP I, stacking interaction between two molecules from chains separated by $3.745 \AA$ has the highest contribution to the total energy with the value of $-35 \mathrm{~kJ} / \mathrm{mol}$ and is dominated by dispersion forces. Slightly lower energy $(-24 \mathrm{~kJ} / \mathrm{mol})$ is observed for the chains separated by $3.940 \AA$. The second strongest interaction $(-32 \mathrm{~kJ} / \mathrm{mol})$ in this crystal structure is the one observed for hydrogen bonded dimers in chains and has mostly electrostatic character.

The opposite situation was observed for the HAP II where two strongest interactions $(-45 \mathrm{~kJ} / \mathrm{mol}$ and $-44 \mathrm{~kJ} / \mathrm{mol})$ were found among hydrogen bonded dimers and have mainly coulombic character. In contrast to HAP I, the stacking interaction between chains of hydrogen bonding is significantly weaker and its total energy equals $-18 \mathrm{~kJ} / \mathrm{mol}$.
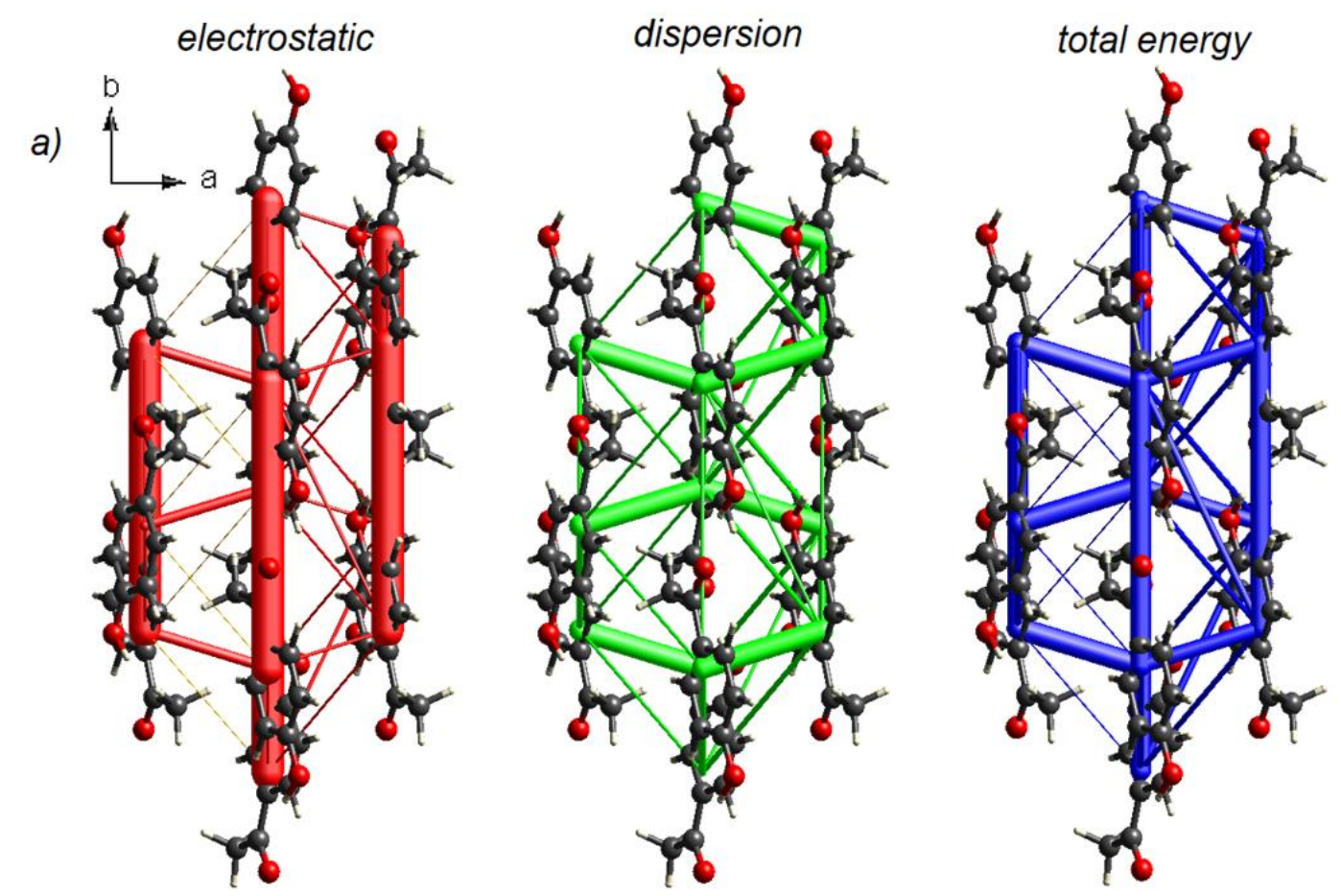

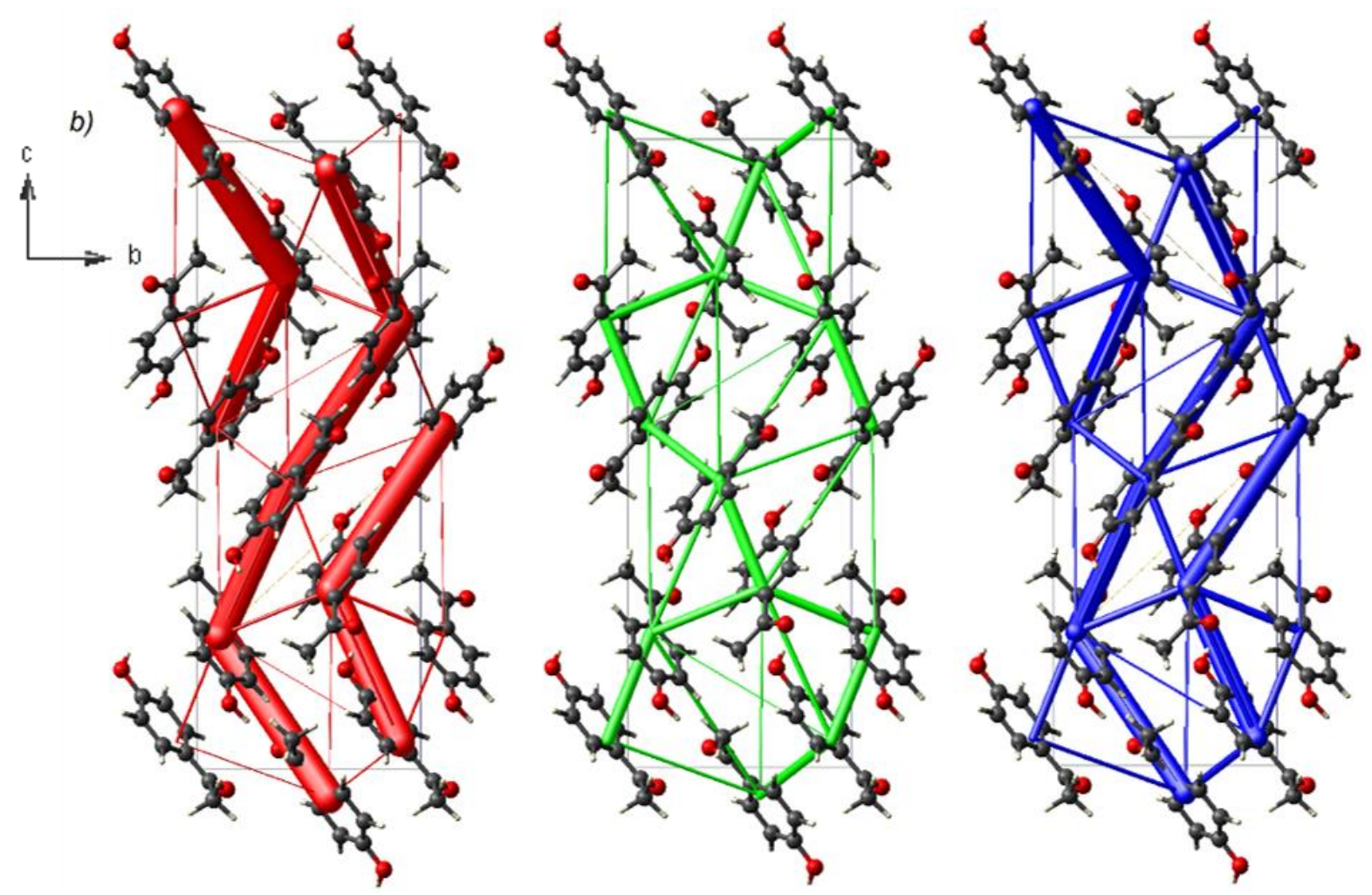

Figure 2. Energy framework diagrams of a) HAP I and b) HAP II polymorphs. Interactions below $1 \mathrm{~kJ} / \mathrm{mol}$ energy threshold have been omitted for the clarity. The tube size was set at 100 for all presented figures enabling direct comparison of the respective dispersion (green) and electrostatic (red) contributions to the total intermolecular energies (blue). 


\subsection{Structures of HAP from multi-temperature measurements}

3.2.1 Structure of HAP I. The structure of HAP form I measured at 40K, $100 \mathrm{~K}, 123 \mathrm{~K}$ and the room temperature $(295 \mathrm{~K})$ stays in agreement with the previously reported structure from $150 \mathrm{~K}$. Structure from $100 \mathrm{~K}$, obtained from neutron diffraction measurements, differ from X-ray structure mainly by X-H bond lengths. The most pronounced differences are observed for $0-\mathrm{H}$...O hydrogen bonding.

While the temperature is raised, as expected, the cell volume increases (from $681 \AA^{3}$ in $40 \mathrm{~K}$ to $714 \AA^{3}$ in $295 \mathrm{~K}$ ). The thermal expansion tensor is plotted in the Figure 3a. We can clearly observe, that cell dimensions a and c are increasing while temperature is increased, whereas in $\mathbf{b}$ direction the situation is opposite. By looking into energy frameworks and by analysing interactions in $\mathbf{a}, \mathbf{b}$ and $\mathbf{c}$ directions, one can easily explain this phenomenon: along $\mathbf{b}$ direction HAP molecules form chains via hydrogen bonding. In this direction interactions are the strongest and the most directional, therefore thermal expansion is negative. Such correlations of thermal expansion of a unit cell with interactions strength in different directions were studied already for many different systems e.g. ${ }^{32,33}$, herein we present how easy it is to correlate thermal expansion with interactions strength in different directions via energy frameworks analysis.

As expected, ADPs are getting larger while the measurement temperature is growing (Figure 3b.) Interestingly, carbon atoms' ADPs on both sides of the ring, although look almost spherical, especially at higher temperatures exhibit slightly different size and orientation - those situated on the same side as oxygen from the acetic group are smaller and elongated in the bond direction, whereas those placed on the other side of the ring perpendicular to the ring plane. 


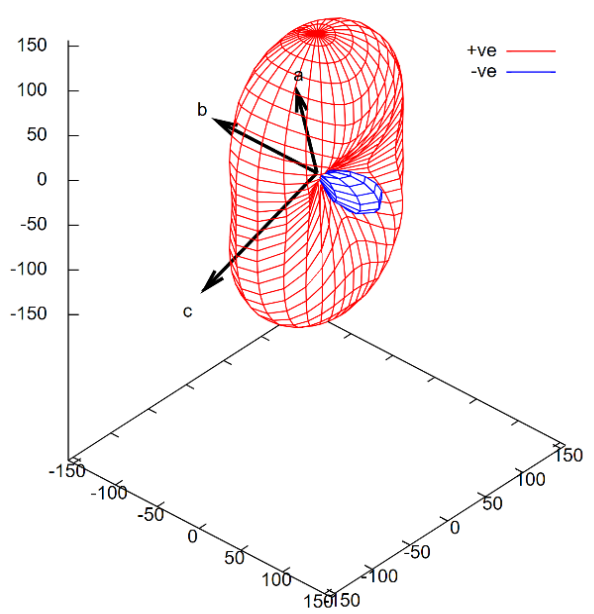

(a)

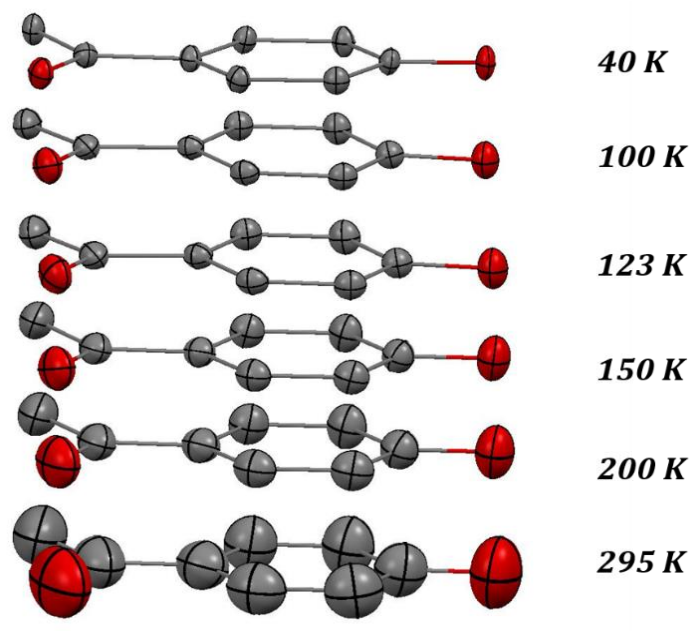

(b)

Figure 3. (a) Unit cell thermal expansion tensor for HAP I (b) comparison of ADPs from different temperatures for HAP I.

3.2.2. HAP II at liquid nitrogen temperature. In the case of HAP II, initial results of diffraction measurements at $123 \mathrm{~K}, 150 \mathrm{~K}, 200 \mathrm{~K}, 300 \mathrm{~K}, 330 \mathrm{~K}$ indicated, as in the case of HAP I, that the structure is identical to the previously known structure. The thermal expansion tensor is completely different in comparison to the one observed for the first polymorph - in the case of HAP II it is negative in $a$ and $c$ directions, and large, positive in b direction. Once again, such shape of thermal expansion tensor can be explained by analysis of intermolecular interactions and energy frameworks. In $b$ direction, structure is the most susceptible for changes, as in this direction the interactions are the weakest: from energy frameworks (Figure 2b), we can clearly state, these chains of hydrogen bonds are affecting $\mathbf{a}$ and $\mathbf{c}$ directions, whereas in $\mathbf{b}$ direction we observe less directional and significantly weaker in this case stacking interactions. In the Figure $\mathbf{4 b}$, we present thermal evolution of ADPs. 


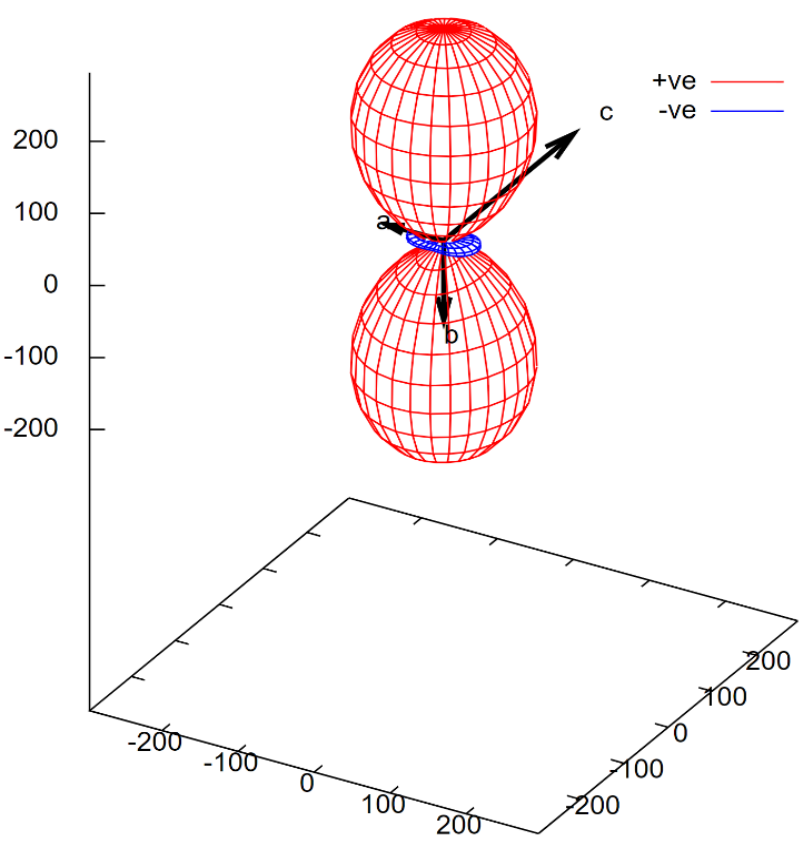

(a)

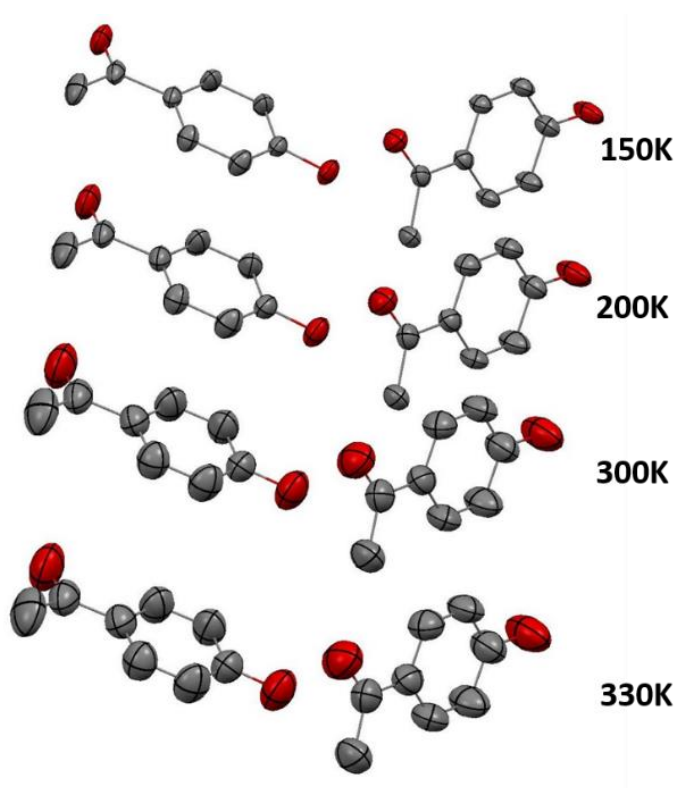

(b)

Figure 4. (a) Unit cell thermal expansion tensor for HAP II (b) comparison of ADPs from different temperatures for HAP II.

At $123 \mathrm{~K}$ following standard integration and data refinement procedures, the data were refined to a reasonable R-factor value of 0.0421 . Routine validation procedure performed with an IUCr checkCIF reported only minor alerts of a C level (namely, large U3/U1 Ratio for Average U(i,j) Tensor). However, after looking at the reconstructed precession images (Figure 5.) it was clear that some additional reflections, which were not observed for this polymorph at $150 \mathrm{~K}$, appear. In the case of $123 \mathrm{~K}$ temperature HAPII even though it was possible to account for additional reflections during data processing and subsequently refine data collected at $123 \mathrm{~K}$ in larger unit cells, none of the proposed solutions led to an unambiguous crystal structure. Therefore, data collection was repeated at $100 \mathrm{~K}$ which allowed us to observe sufficient intensity to sigma ratio of additional peaks.

Such small satellite spots in the diffraction pattern suggest that the structure is modulated. What is the difference between a modulated structure and a perfect crystal? A perfect crystal is built by repeating the unit cell by translation along the three directions of space. In the case of modulated structures, at least in one direction, an additional periodic variation of the atomic sites (positions and/or occupancies and/or ADPs) with respect to an ideal average periodic structure occurs. When the periodicity of this 
modulation is commensurate with lattice parameters, a structure can be solved either in supercell, with larger cell and higher Z' than the average structure, or by using so-called superspace approach - with a small unit cell and modulation vector (see Figures $\mathbf{5}$ and 6).

The new, HAPII 100K structure appears to be commensurately modulated (abbrev. cmHAPII). It can be indexed in a new, larger unit cell (a=9.0155 $\AA, \mathbf{b}=24.4861 \AA$, $\mathbf{c}=30.9055 \AA$ ) with 10 independent molecules in the asymmetric unit. Neutron diffraction measurements at $100 \mathrm{~K}$ confirmed large unit cell (cell parameters $\mathbf{a}=8.9946+$ /- 0.0067 $\AA \mathbf{b}=24.4806+/-0.0167 \AA \mathbf{c}=30.9195+/-0.0176 \AA$ ), however we were not able to solve a structure based on the neutron data. Thus, below we are presenting results from refinements against X-ray data.
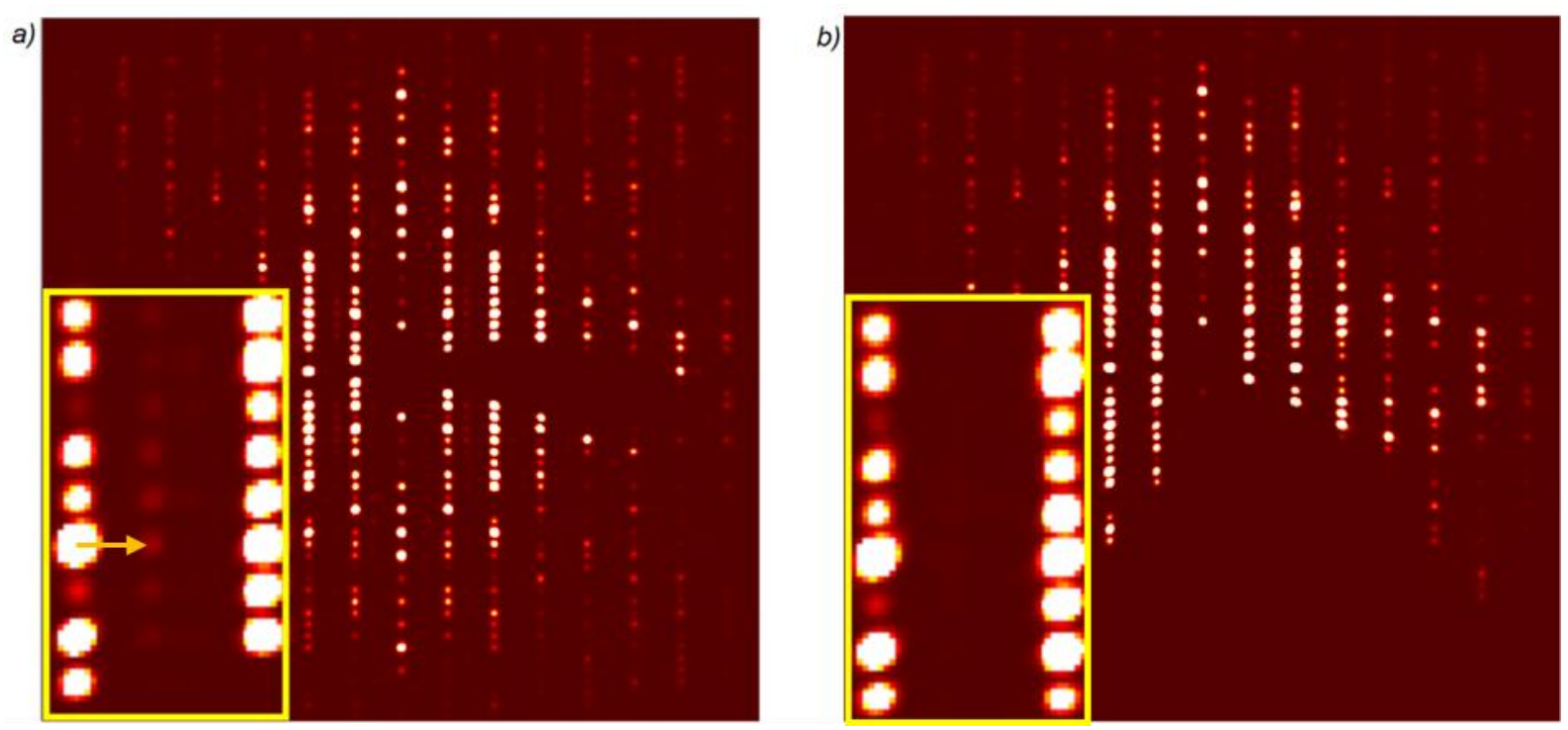

Figure 5. Reconstructed reciprocal h0l layers of the a) HAP II at $123 \mathrm{~K}$ and b) HAP II at $150 \mathrm{~K}$. Notice additional reflections at $123 \mathrm{~K}$ between main reflections which are not present at $150 \mathrm{~K}$. 


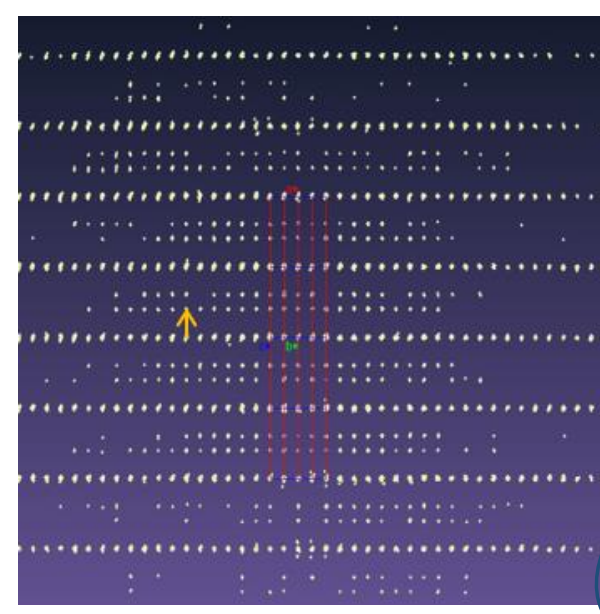

(a)

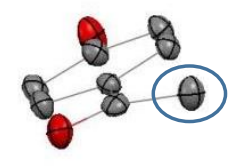

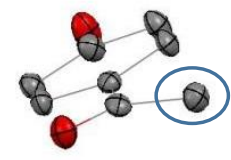

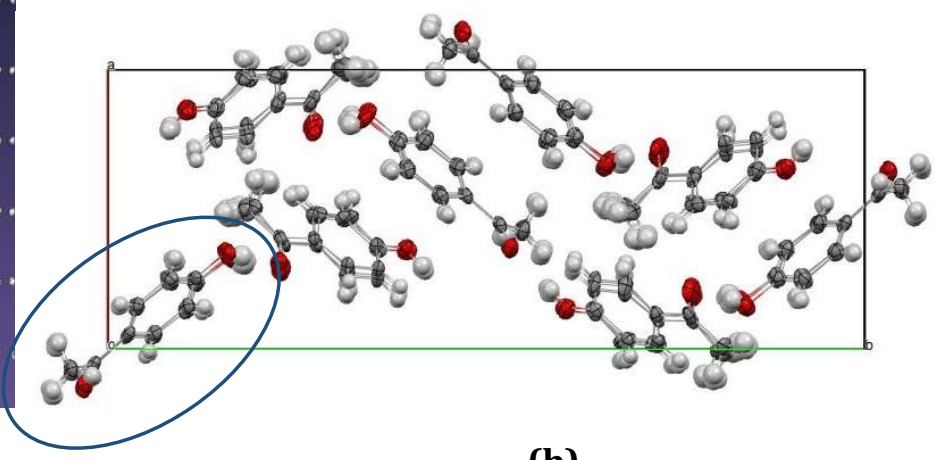

(b)
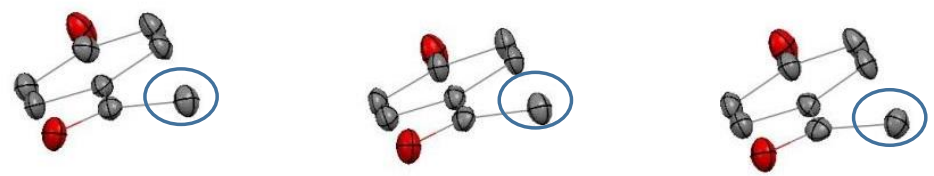

(c)

Figure 6. cmHAPII structure: (a) reconstruction of Ewald sphere viewed along the $\mathrm{b}^{*}-$ visible small additional satellite peaks (b) view of unit cell in the [001] direction (c) HAP molecules from asymmetric part of the unit cell view along the $c$ axis

cmHAPII can be described as a supercell solution of higher temperature HAP II structure with five times more molecules and five times bigger cell volume than the base structure. As a result, the structure has a $Z^{\prime}=10$. Description of ADPs in the structure is significantly improved as many more parameters are considered (Figure 6 c.)

Alternatively, the commensurately modulated structure was solved by SuperFlip ${ }^{34}$ in the space group $\mathrm{P} 2{ }_{1} 2{ }_{1} 2_{1}(\sigma 00)$ with the modulation wave vector $\mathrm{q}$ in the superspace model of $\mathrm{q}=2 / 5 \mathrm{a}^{*}$ and cell parameters $\mathbf{a}=6.1800 \AA, \mathbf{b}=9.0200 \AA$ and $\mathbf{c}=24.5060 \AA$. Note, owing to the standard choice of the unit cell in the orthorhombic space group the order of the base vector changes from (abc) to (bca). To describe the modulation, in the final model continuous atomic modulation functions for displacive modulation and modulation of the anisotropic ADPs (with higher harmonic up to $2^{\text {nd }}$ order) are applied to all non-hydrogen atoms. The hydrogen atoms are coupled via a riding model to their corresponding carbon/oxygen atoms. 


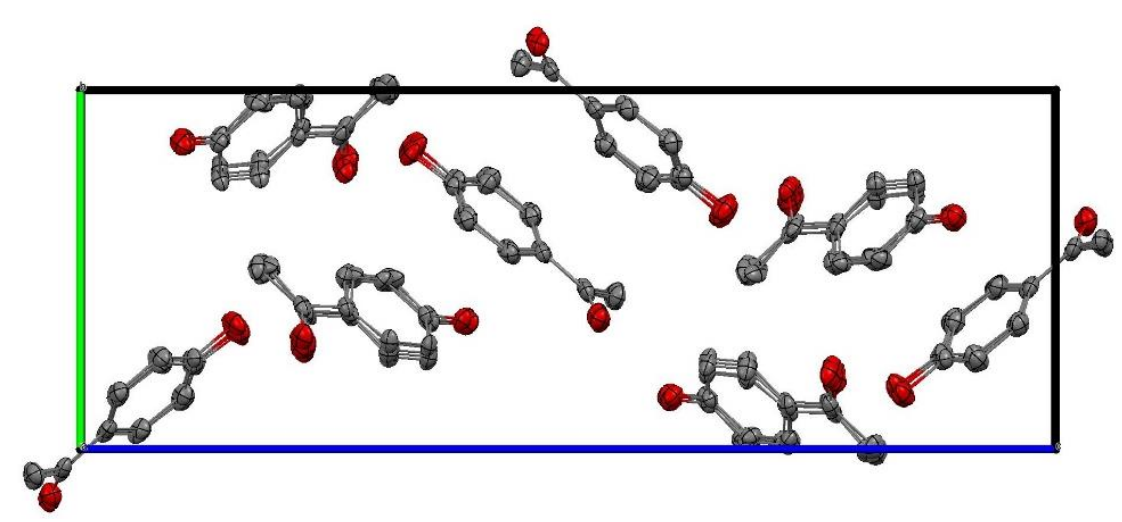

(a)

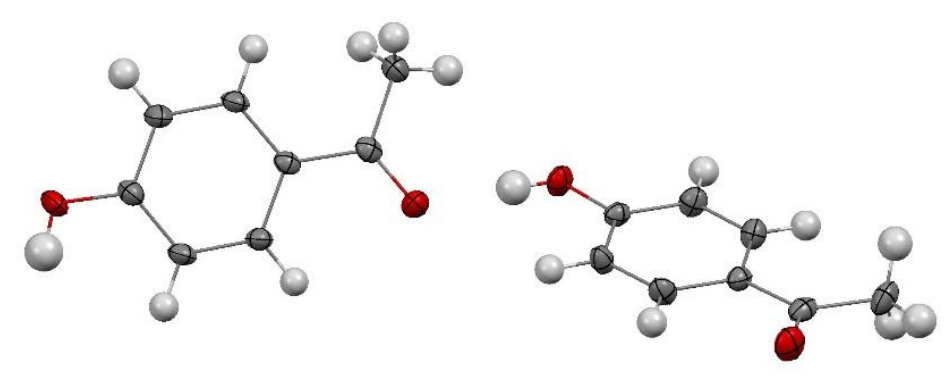

(b)

Figure 7. Solution with superspace approach. (a) approximant structure - similar to $Z^{\prime}=10$ structure, (b) averaged structure $-A D P$ s similar to $Z^{\prime}=2$ structure. See also attached movie (hap_100K.avi)

3.2.3. HAP II at liquid helium temperature. Further lowering of temperature reveal that at $40 \mathrm{~K}$, the HAP II structure has even more complex nature. At $40 \mathrm{~K}$, in addition to satellite peaks observed along $\mathrm{a}^{*}$ axis, we observed a new set of reflections in the $\mathrm{b}^{*}$ direction. Main reflections are accompanied by first and second order satellite reflections.

In this direction, periodicity of modulation is not commensurate with cell parameters -it means, that structure is incommensurately modulated (abbrev. imHAPII) and can be solved only by using the superspace approach. It was possible to solve the structure in the monoclinic $\mathrm{P} 2{ }_{1}(\sigma \gamma 0)$ space group with a modulation wave vector $\mathrm{q}=[0.38,0.17,0.0]$ and 4 symmetry-independent molecules in the asymmetric unit. Cell parameters remain close to the commensurate structure with: $a=6.1738(7) \AA, b=8.9627(10) \AA ̊, c=24.516(3) \AA \AA$, 


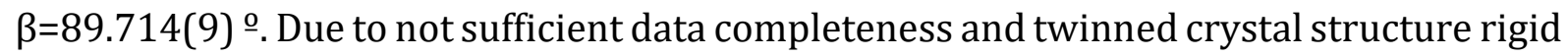
body approach was executed to diminish the number of refined parameters. Rigid body position was described with continuous displacive modulation of rotation and translation. Additionally, ADPs were derived from TLS rigid-body analysis with two modulation function for tensor components of translation, libration and screw-rotation.

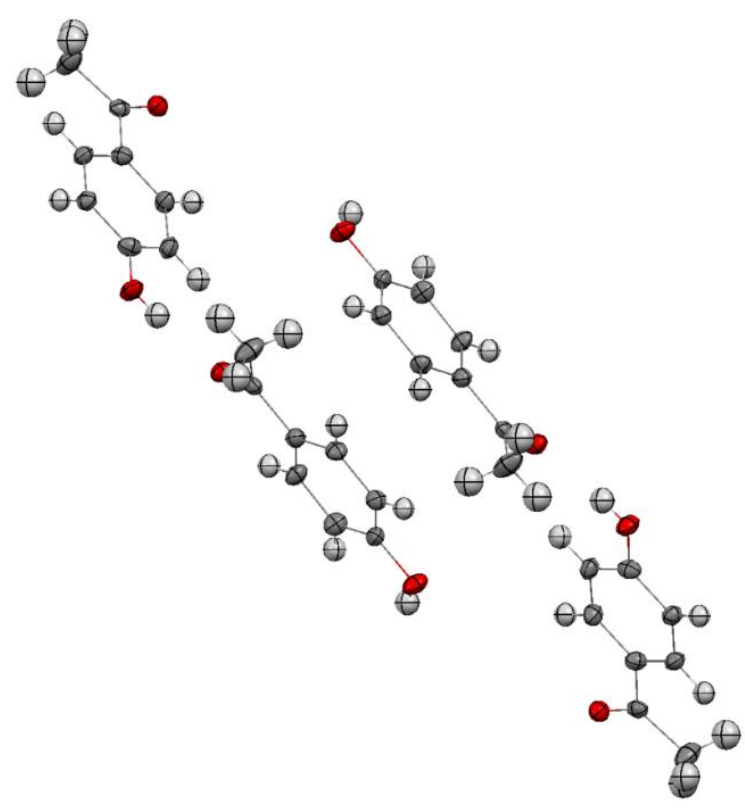

Figure 8. Representation of the averaged structure of HAP II at $40 \mathrm{~K}$ viewed along $a$-axis. See also attached movie (hap_40K.avi)

\section{Energy calculations results}

According to earlier investigations of relative stability of polymorphic forms of hydroxyacetophenone the thermodynamically stable form at low temperature is orthorhombic form, HAP II. Theoretical periodic ab-initio calculations, which we conducted are in good agreement with experimental results from calorimetry: they indicate HAP II as the more stable form (by c.a. $2-3 \mathrm{~kJ} / \mathrm{mol}$ ). As the phase transition from HAP II to HAP I is entropy driven, and our calculations take into account only enthalpy, we did not observe changes in stability order at room temperature.

We wanted to check, whether it will make a difference, if we use for our calculations as a starting point structure of the orthorhombic form, which contains ten molecules in the asymmetric unit, or the orthorhombic structure of HAP II with two molecules in 
asymmetric unit. Surprisingly, obtained lattice and total energies for both Z'=2 and Z'=10 structures are almost the same.

Table 1. Total energy per molecule and lattice energy in the brackets. All values are in $\mathrm{kJ} / \mathrm{mol}$

\begin{tabular}{lrrrr}
\hline $\begin{array}{r}\text { Computational } \\
\text { method }\end{array}$ & \multicolumn{1}{c}{ PBE0-D3 } & PBE0-D2 & \multicolumn{1}{c}{$\begin{array}{c}\text { B3LYP- } \\
\text { D3ABC/VTZ/HF-3c }\end{array}$} & $\begin{array}{c}\text { B3LYP-D3ABC/6- } \\
\text { 31/HF-3c }\end{array}$ \\
\hline HAP I & -1207106.22 & -1207102.80 & -1207790.94 & -1207574.80 \\
& $(-119.9)$ & $(-133.6)$ & $(-113.7)$ & \\
HAP II (Z'=2) & -1207109.18 & -1207105.07 & -1207794.50 & -1207579.51 \\
& $(-127.5)$ & $(-138.9)$ & $(-119.5)$ & \\
HAP II (Z'=10) & - & - & -3.56 & -1207579.48 \\
\hline$\Delta \mathrm{E}_{\text {HAPI-HAPII }}$ & -2.96 & -2.27 & $(-5.9)$ & -4.71 \\
& $(-7.6)$ & $(-5.3)$ & - & -0.03
\end{tabular}

Therefore, one may raise a question: as energies for the orthorhombic form of hydroxyacetophenone with two molecules in asymmetric unit (asu) remains the same as for ten molecules in asu, why do we observe a modulation at low temperature? In the case of modulated systems, very often modulation can be rationalised as result of a compromise between the steric demands of the molecule and the optimal arrangement of hydrogen bonding moieties ${ }^{35}$. We decided to investigate dimer interaction energies, and verify, whether due to modulation some interactions for the structure with $Z^{\prime}=10$ are becoming significantly stronger than in the case of averaged structure, with $Z^{\prime}=2$.

From our analysis of dimer interactions energies (see Figure 9) we conclude that for two types of hydrogen bonds energies are almost the same as in the case of HAP II with Z'=2. In the case of $Z^{\prime}=10$ structure energies for first type of hydrogen bond vary between -42.5 and $-43.1 \mathrm{~kJ} / \mathrm{mol}$ and for the second type between -41.8 and $-42.4 \mathrm{~kJ} / \mathrm{mol}$, and on average they are equal to energies obtained for $\mathrm{Z}^{\prime}=2$ structure $(-42.7$ and $-42.0 \mathrm{~kJ} / \mathrm{mol})$. We observe larger deviations from average for weak dispersive interactions (green energy values on Figure 9). Weak dispersive interactions between parallel 
hydroxyacetophenone molecules vary between $-11.6 \mathrm{~kJ} / \mathrm{mol}$ up to $-13 \mathrm{~kJ} / \mathrm{mol}$. We may conclude that locally, some of those week interactions are stronger than in the case of Z'=2 structure, but on the other hand some are weaker, and on average structures with $Z^{\prime}=2$ and $Z^{\prime}=10$ have almost the same electronic energy.

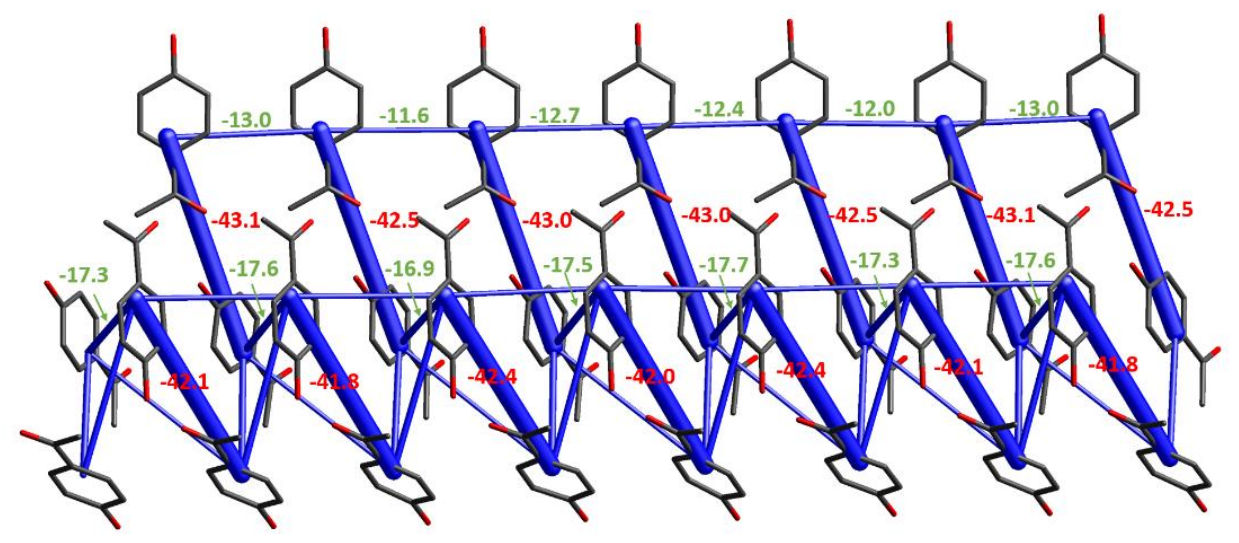

(a)

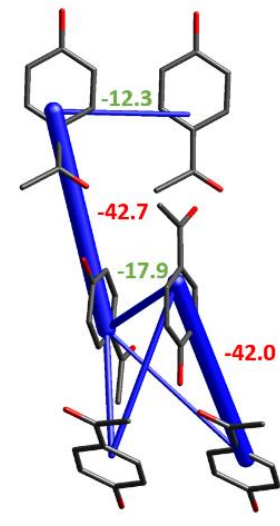

(b)

Figure 9. Comparison of energy frameworks for $Z^{\prime}=10$ (a) and $Z^{\prime}=2$ (b) structures.

Interesting discussion of similar cases, for which high temperature phase has lower Z' than the low temperature phase was presented by Steed \& Steed ${ }^{36}$. For example, similarly to our case, Tröger's base derivative, undergoes a $Z^{\prime}=1$ to $Z^{\prime}=3$ phase transition on cooling below $130 \mathrm{~K}^{37}$. The authors suggest, that although the higher Z' structure exhibit lower energy, the more stable at higher temperatures is the $Z^{\prime}=1$ structure due to increase of entropic term with the temperature. Similarly, in our case, entropic term most probably is stabilizing structure with $Z^{\prime}=1$.

\section{Importance of ADPs: computed vs experimental ADPs}

Experimental ADPs are prone to all experimental errors. A simple test, which should show, whether ADPs are correct, is the Hirshfeld rigid bond test. It tests whether the components of the anisotropic displacement parameters along chemical bonds are equal in magnitude. Large differences might indicate a systematic distortion of these parameters with other (unresolved) effects such as (substitutional) disorder, model or data errors and/or over-refinement. Atomic sites assigned the wrong scattering type (e.g. $\mathrm{Ag}$ versus $\mathrm{Br}$ ) should generate 'problem signals' with this test. Data sets corrected for 
absorption effects with DELREF techniques (e.g. DIFABS, SHELXA, XABS2) often show large DELU values for bonds involving the heaviest atom.

Interestingly, in the case of both polymorphic forms of HAP at $123 \mathrm{~K}$, the Hirshfeld rigid bond test is fulfilled. However, a closer look into ADPs of HAP II showed strange features that convince as to conduct theoretical computations of ADPs for both HAP forms to compare results. Due to fast increase in computer power, calculation of ADPs for molecular crystal started to be feasible. Usually, computed ADPs are compared with experimental once (preferably from neutron diffraction experiments), in order to verified quality of theoretical calculations ${ }^{31,38,39 .}$

Herein, we would like to show, that computation of theoretical ADPs to compare them to experimental one can pinpoint some still unresolved features in the crystal structure. Without doubt, special attention should be always be paid to the experimental diffraction data.

As ADPs are a mean value over the Brillouin zone, and those computed by us are based only on $\Gamma$ point frequencies, computed ADPs would not be exactly equal to experimental ADPs. However, some features of ADPs should be preserved, and in the case of HAP I main trends are preserved - on one side of the ring ADPs are elongated in a direction perpendicular to the ring plane, whereas on the other structure, in bond direction.

From theoretical computations we even can indicate one particular vibration with low frequency $\left(80 \mathrm{~cm}^{-1}\right)$ that is responsible for such shape of aromatic carbon atom ADPs. In the case of HAP II computed ADPs differ significantly from experimental ones (see Figure 10 b). Experimental ADPs, for one of the HAP molecules, are elongated in one direction, and for the second molecule ADPs from ring seems to follow libration around C1...C4 axis. None of those features is observed in the case of computed ADPs.

Taking into account satellite peaks and, as consequence, modulation of the HAP II structure in $100 \mathrm{~K}$ results in ADPs for averaged structure very similar to those from $150 \mathrm{~K}$. Thus, we may conclude that $\mathrm{Z}^{\prime}=2$ high temperature structure is an average structure that does not fully account for all of the small conformational differences in HAP molecule geometry in the $\mathrm{Z}^{\prime}=10$ system. Similar conclusion was made for another interesting example, namely the complex $[\mathrm{Ni}(\mathrm{OAc})(\mathrm{PNPtBu})] \mathrm{OT}$, which contains one independent molecule in the asymmetric unit of the crystal structure with very large anisotropic 
displacement parameters, and undergoes phase transition on cooling to low-temperature phase, which has six independent, well ordered molecules in the asymmetric unit 40 .
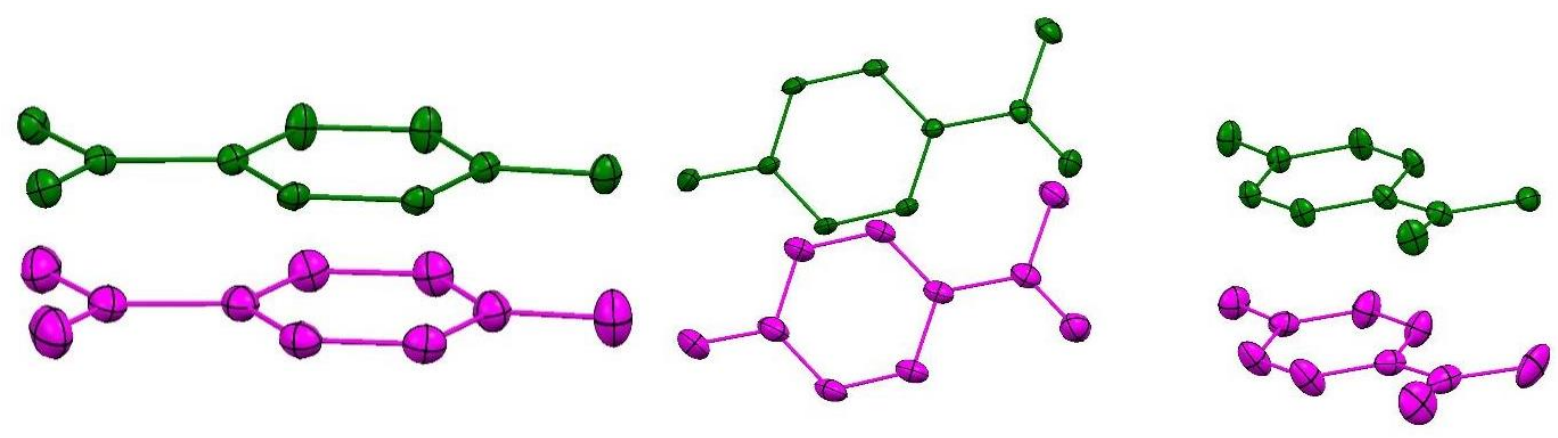

(a)

(b)

Figure 10. Anisotropic displacement parameters computed (top, green) vs experimental (bottom, magenta) (a) HAP I (b) HAP II.

\section{Conclusions}

In this contribution we present, that even such simple and well-studied structure of 4'hydroxyacetophenone, when studied with low and ultra-low temperatures, can reveal a more complex nature. After cooling of orthorhombic form II of 4'-hydroxyacetophenone to $100 \mathrm{~K}$, we obtain commensurately modulated structure, with five times more molecules in the asymmetric unit than the room temperature structure. By further lowering temperature to $40 \mathrm{~K}$, the structure is becoming even more complicated, incommensurately modulated. This structure is thermodynamically stable at low temperatures as confirmed by our theoretical investigations. Consideration of additional diffraction spots and superstructure with $Z^{\prime}=10$ indicated the same lattice (or bulk) energy as $Z^{\prime}=2$ structure. It was confirmed by detailed analysis of dimer interaction energies, that in $\mathrm{Z}^{\prime}=10$ structure some dimer interactions are stronger than in the case of Z'=2 structure influencing weaker ones and on average structures exhibit almost the same electronic energy. Therefore, most probably, phase transition (HAPII > HAP I) is driven by entropy. Additionally, it is important to remember, that in the case of HAP polymorphs, as conformation of both HAP molecules for forms I and II is different, kinetics also might play a role. 
Both structures form head-to-tail hydrogen bonds and stacking interactions that were quantified with the aid of the energy frameworks analysis. Stable at low-temperature polymorph (HAP II) forms stronger hydrogen bond and weaker stacking interactions by the means of lower energy syn molecular conformation. On the contrary, the HAP I case exemplifies opposite features. Both structures illustrate a compromise between the dispersive and electrostatic forces to achieve a stable structure. Additionally, energy frameworks can help with understanding strength of interactions in given directions, and in consequence, the shape of thermal expansion tensors - indeed, in directions in which interactions are weaker thermal expansion is larger than in the case of directions in which interactions are stronger.

Comparison of experimentally obtained and computed ADPs reveals significant differences between ADPs in the case of HAP II. It is not an indication that theory is wrong, but it might be also that some more attention should be put to experimental data. In general, we recommend careful analyse of ADPs especially in the case of polymorphic systems as they can behave as dustbin to all experimental errors, therefore rationalization and explanation of their size and orientation are crucial for correct data analysis. In such a situation, conducting multi-temperature measurements, observing temperature evolution of ADPs and going to low and ultra-low temperatures is vital for understanding the complex solid-state chemistry. Especially in the view that polymorph relative stability, modulated and high Z' structures are still a challenge for theoretical methods and for crystal structure prediction.

Additionally, it is worth to remember, that the lattice energy is computed for an ideal crystal, without any disorders, incommensurate modulations, etc. Therefore, computed values might not always agree with experimental ones. Thus, in order to progress in computational sciences, not only good new functionals and dispersion corrections are needed, but also state-of-the-art low-temperature measurements! Moreover, as all data deposited in the CSD might be later utilised by other researchers, it is of great importance, even when X-ray data collection was done just to confirm the structure, to give as many details about a measurement as possible and to report all problems which occurred during structure refinement. 


\section{Supporting Information}

Supporting Information contains crystallographic details for HAP I and HAP II after TAAM refinements, the de Wolf sections for commensurate and incommensurate HAP II structure, neutron data collection details. This material is available free of charge via the Internet at http://pubs.acs.org.

\section{Acknowledgements}

M.S. and A.A.H. would like to thank the Foundation for Polish Science for founding within HOMING Programme (Homing/2016-1/3 grant), financed by the European Union under the European Regional Development Fund (POIR.04.04.00-00-1DAE/16-00). This research was supported in part by PL-Grid Infrastructure. ISIS, experiment number RB1720131 is kindly acknowledged. Authors would like to thank to dr Michal Dusek for his help with X-ray data integration for the incommensurate structure. The Jana software is developed under Project No. 18-10504S of the Czech Science Foundation

\section{References:}

(1) Cruz-Cabeza, A. J.; Reutzel-Edens, S. M.; Bernstein, J., Facts and fictions about polymorphism. Chem. Soc. Rev. 2015, 44, 8619-8635.

(2) Nyman, J.; Day, G. M., Static and lattice vibrational energy differences between polymorphs. CrystEngComm 2015, 17, 5154-5165.

(3) Jarzembska, K. N.; Dominiak, P. M., New version of the theoretical databank of transferable aspherical pseudoatoms, UBDB2011 - towards nucleic acid modelling. Acta Crystallogr., Sect. A 2012, 68, 139-147.

(4) Dominiak, P. M.; Volkov, A.; Li, X.; Messerschmidt, M.; Coppens, P., A Theoretical Databank of Transferable Aspherical Atoms and Its Application to Electrostatic Interaction Energy Calculations of Macromolecules. J. Chem. Theory Comput 2007, 3, 232-247.

(5) Jayatilaka, D.; Dittrich, B., X-ray structure refinement using aspherical atomic density functions obtained from quantum-mechanical calculations. Acta Crystallogr., Sect. A 2008, 64, 383393.

(6) Capelli, S. C.; Burgi, H.-B.; Dittrich, B.; Grabowsky, S.; Jayatilaka, D., Hirshfeld atom refinement. IUCrJ 2014, 1, 361-379.

(7) Fugel, M.; Jayatilaka, D.; Hupf, E.; Overgaard, J.; Hathwar, V. R.; Macchi, P.; Turner, M. J.; Howard, J. A. K.; Dolomanov, O. V.; Puschmann, H.; Iversen, B. B.; Burgi, H.-B.; Grabowsky, S., Probing the accuracy and precision of Hirshfeld atom refinement with HARt interfaced with Olex2. IUCrJ 2018, 5, 32-44.

(8) Bond, A. D., Pharmaceutical crystallography: is there a devil in the details? CrystEngComm 2012, 14, 2363-2366.

(9) Hoser, A. A.; Sovago, I.; Lanza, A.; Madsen, A. Ø., A crystal structure prediction enigma solved: the gallic acid monohydrate system - surprises at $10 \mathrm{~K}$. Chem. Commun. 2017, 53, 925-928.

(10) Bernardes, C. E. S.; Piedade, M. F. M.; Piedade, M. E. M. d., Polymorphism in 4'Hydroxyacetophenone: Structure and Energetics. Cryst. Growth Des. 2008, 8, 2419-2430.

(11) Joseph, A.; Bernardes, C. E. S.; Druzhinina, A. I.; Varushchenko, R. M.; Nguyen, T. Y.; Emmerling, F.; Yuan, L.; Dupray, V.; Coquerel, G.; da Piedade, M. E. M., Polymorphic Phase Transition 
in 4'-Hydroxyacetophenone: Equilibrium Temperature, Kinetic Barrier, and the Relative Stability of Z' = 1 and $Z^{\prime}=2$ Forms. Cryst. Growth Des. 2017, 17, 1918-1932.

(12) Kulkarni, S. A.; Meekes, H.; ter Horst, J. H., Polymorphism Control through a Single Nucleation Event. Cryst. Growth Des. 2014, 14, 1493-1499.

(13) CrysAlis CCD and CrysAlis RED, Oxford Diffraction, Oxford Diffraction Ltd, Yarnton 2008.

(14) Sheldrick, G. M., A short history of SHELX. Acta Crystallogr., Sect. A 2008, 64, 112-122.

(15) Petříček, V.; Dušek, M.; Palatinus, L., Crystallographic Computing System JANA2006: General features. In Z. Kristallogr., 2014; 229, 345-352.

(16) Sanjuan-Szklarz, W. F.; Hoser, A. A.; Gutmann, M.; Madsen, A. O.; Wozniak, K., Yes, one can obtain better quality structures from routine X-ray data collection. IUCrJ 2016, 3, 61-70.

(17) Jelsch, C.; Guillot, B.; Lagoutte, A.; Lecomte, C., Advances in protein and small-molecule charge-density refinement methods using MoPro. J. Appl. Crystallogr. 2005, 38, 38-54.

(18) Cliffe, M. J.; Goodwin, A. L., PASCal: a principal axis strain calculator for thermal expansion and compressibility determination. J. Appl. Crystallogr. 2012, 45, 1321-1329.

(19) Keen, D. A.; Gutmann, M. J.; Wilson, C. C., SXD - the single-crystal diffractometer at the ISIS spallation neutron source. J. Appl. Crystallogr. 2006, 39, 714-722.

(20) Gutmann, M. J. SXD2001, ISIS, Rutherford Appleton Laboratory, Oxfordshire, England: 2001.

(21) Mackenzie, C. F.; Spackman, P. R.; Jayatilaka, D.; Spackman, M. A., CrystalExplorer model energies and energy frameworks: extension to metal coordination compounds, organic salts, solvates and open-shell systems. IUCrJ 2017, 4, 575-587.

(22) Turner, M. J.; Thomas, S. P.; Shi, M. W.; Jayatilaka, D.; Spackman, M. A., Energy frameworks: insights into interaction anisotropy and the mechanical properties of molecular crystals. Chem. Commun. 2015, 51, 3735-3738.

(23) Dovesi, R.; Saunders, V. R.; Roetti, C.; Orlando, R.; Zicovich-Wilson, C. M.; Pascale, F.; Civalleri, B.; Doll, K.; Harrison, N. M.; Bush, I. J.; D’Arco, P.; Llunell, M.; Causà, M.; Noël, Y.; Maschio, L.; Erba, A.; Rerat, M.; Casassa, S. CRYSTAL17 User's Manual, University of Torino, Torino., 2017.

(24) Dovesi, R.; Erba, A.; Orlando, R.; Zicovich-Wilson, C. M.; Civalleri, B.; Maschio, L.; Rérat, M.; Casassa, S.; Baima, J.; Salustro, S.; Kirtman, B., Quantum-mechanical condensed matter simulations with CRYSTAL. . WIREs Comput Mol Sci 2018, e1360.

(25) Adamo, C.; Barone, V., Toward reliable density functional methods without adjustable parameters: The PBE0 model. J. Chem. Phys. 1999, 110, 6158-6170.

(26) Schäfer, A.; Huber, C.; Ahlrichs, R., Fully optimized contracted Gaussian basis sets of triple zeta valence quality for atoms Li to Kr. J. Chem. Phys. 1994, 100, (8), 5829-5835.

(27) Grimme, S.; Antony, J.; Ehrlich, S.; Krieg, H., A consistent and accurate ab initio parametrization of density functional dispersion correction (DFT-D) for the 94 elements H-Pu. J. Chem. Phys. 2010, 132, 154104.

(28) Grimme, S.; Ehrlich, S.; Goerigk, L., Effect of the damping function in dispersion corrected density functional theory. J. Comput. Chem. 2011, 32, 1456-1465.

(29) Cutini, M.; Civalleri, B.; Corno, M.; Orlando, R.; Brandenburg, J. G.; Maschio, L.; Ugliengo, P., Assessment of Different Quantum Mechanical Methods for the Prediction of Structure and Cohesive Energy of Molecular Crystals. J. Chem. Theory Comput. 2016, 12, 3340-3352.

(30) Lee, C. R.; Yang, W.; Parr, R. G., Phys. Rev. B 1988, 37, 785.

(31) Madsen, A. O.; Civalleri, B.; Ferrabone, M.; Pascale, F.; Erba, A., Anisotropic displacement parameters for molecular crystals from periodic Hartree-Fock and density functional theory calculations. Acta Crystallogr., Sect. A 2013, 69, 309-321.

(32) Bhattacharya, S.; Saha, B. K., Interaction Dependence and Similarity in Thermal Expansion of a Dimorphic 1D Hydrogen-Bonded Organic Complex. Cryst.l Growth Des. 2013, 13, 3299-3302.

(33) Durka, K.; Hoser, A. A.; Kamiński, R.; Luliński, S.; Serwatowski, J.; Koźmiński, W.; Woźniak, K., Polymorphism of a Model Arylboronic Azaester: Combined Experimental and Computational Studies. Cryst. Growth Des. 2011, 11, 1835-1845

(34) Palatinus, L.; Chapuis, G., SUPERFLIP- a computer program for the solution of crystal structures by charge flipping in arbitrary dimensions. J. Appl. Crystallogr. 2007, 40, 786-790. 
(35) Schönleber, A., Organic molecular compounds with modulated crystal structures. $Z$. Kristallogr. 2011, 226, 499-517.

(36) Steed, K. M.; Steed, J. W., Packing Problems: High Z' Crystal Structures and Their Relationship to Cocrystals, Inclusion Compounds, and Polymorphism. Chem. Rev. 2015, 115, 2895-2933.

(37) Vande Velde, C. M. L.; Tylleman, B.; Zeller, M.; Sergeyev, S., Structures of alkyl-substituted Troger's base derivatives illustrate the importance of $Z^{\prime}$ for packing in the absence of strong crystal synthons. Acta Crystallogr., Sect. B 2010, 66, 472-481.

(38) Deringer, V. L.; Stoffel, R. P.; Togo, A.; Eck, B.; Meven, M.; Dronskowski, R., Ab initio ORTEP drawings: a case study of $\mathrm{N}$-based molecular crystals with different chemical nature. CrystEngComm 2014, 16, 10907-10915.

(39) Erba, A.; Ferrabone, M.; Orlando, R.; Dovesi, R., Accurate dynamical structure factors from ab initio lattice dynamics: The case of crystalline silicon. J. Comput. Chem. 2013, 34, 346-354.

(40) Lutz, M.; van der Vlugt, J. I.; Vogt, D.; Spek, A. L., A reversible solid-solid phase transition Z'=1 to $Z^{\prime}=6$ in $[N i(O A c)(P N P t B u)] O T f$. Polyhedron 2009, 28, 2341-2346. 


\section{For Table of Contents Use Only}

"How accurate $X$-ray data are needed to obtain reliable order of stability for polymorphs? The case study of p-hydroxyacetophenone polymorphs."

Marcin Sztylko, Maura Malinska, Vaclav Petricek, Matthias J. Gutmann, Anna A. Hoser

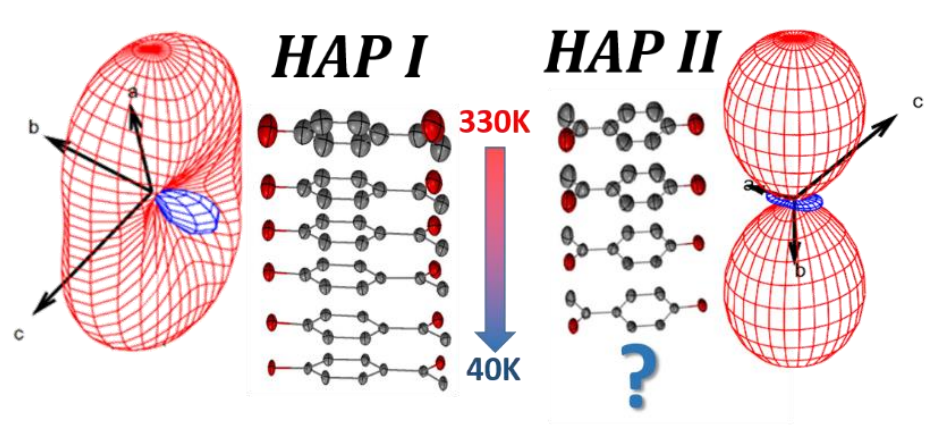

The importance of high quality, low-temperature X-ray diffraction experiments is discussed based on the new, modulated structures found for polymorph of 4'-hydroxyacetophenone (HAP) 